\title{
Objective image analysis of non-magnifying image-enhanced endoscopy for diagnosis of small depressed early gastric
} cancers

\section{다(1)이우}

\author{
Authors \\ Jiro Watari ${ }^{1}$, Masaya Kobayashi ${ }^{1}$, Keisuke Nakai ${ }^{1}$, Chiyomi Ito ${ }^{1}$, Akio Tamura ${ }^{1}$, Tomohiro Ogawa ${ }^{1}$, Takahisa Yamasaki ${ }^{1}$, \\ Takuya Okugawa', Takashi Kondo', Tomoaki Kono', Katsuyuki Tozawa', Toshihiko Tomita', Tadayuki Oshima', \\ Hirokazu Fukui ${ }^{1}$, Takeshi Morimoto ${ }^{2}$, Hiroto Miwa ${ }^{1}$
}

Institutions

1 Division of Gastroenterology, Department of Internal Medicine, Hyogo College of Medicine, Nishinomiya, Japan

2 Department of Clinical Epidemiology, Hyogo College of Medicine, Nishinomiya, Japan

submitted 17.4 .2018

accepted after revision 31.7 .2018

\section{Bibliography}

DOI https://doi.org/10.1055/a-0735-9317 |

Endoscopy International Open 2018; 06: E1445-E1453

(c) Georg Thieme Verlag KG Stuttgart · New York

ISSN 2364-3722

\section{Corresponding author}

Jiro Watari, MD PhD, Division of Gastroenterology, Department of Internal Medicine, Hyogo College of Medicine, 1-1, Mukogawa-cho, Nishinomiya, Hyogo 6638501, Japan

Fax: +81-798-456661

watarij@hyo-med.ac.jp

\section{ABSTRACT}

Background Gastric cancers (GC) after $H$. pylori eradication are difficult to diagnose even by magnifying narrowband imaging (NBI) or blue laser imaging (BLI) endoscopy. Little is known with regard to non-magnifying (NM)-NBI/
BLI for early GC so we examined the efficacy of NM-NBI/BLI for early GC diagnosis.

Methods We retrospectively analyzed the images of 29 small $(\leq 1 \mathrm{~cm})$ intramucosal GC that had been treated with endoscopic submucosal dissection and 137 benign depressed lesions (BDLs). The brightness and shape of the GCs and BDLs by NM-NBI/BLI were assessed with Image software.

Results The NBI/BLI-index, which indicates the brightness of NBI/BLI for visualization, was significantly higher in GC than BDLs in both the $H$. pylori-infected $(P=0.009)$ and -eradicated group $(P<0.0001)$, indicating that GC exhibited brighter colors than the normal surrounding mucosa. The C-index, which refers to the circularity of the lesion, was also significantly higher in GC than BDLs in both $\mathrm{H}$. pylori-infected $(P=0.006)$ and -eradicated cases $(P=0.004)$. Based on receiver-operating characteristic curve analysis, cutoff values for the NBI/BLI- and C-indices for GC were 1.04 and 0.58 in the $H$. pylori-infected cases, and 0.98 and 0.64 in the $H$. pylori-eradicated cases. With the reference value of the $\mathrm{NBI} / \mathrm{BLI}$-index set at $\geq 0.69$ with the $\mathrm{C}$-index at $\geq 0.21$ in the $H$. pylori-infected and the NBI/BLI-index at $\geq 0.80$ with the $C$ index at $\geq 0.32$ in the $H$. pylori-eradicated cases, both the sensitivity and negative predictive value for early GC were $100 \%$. A high NBI/BLI-index tended to be associated with a wide length of the intervening part histologically in the $H$. pylori-eradicated cases $(P=0.09)$.

Conclusions The small depressed-type early GC had brighter color and rounder shape compared to BDLs in both $H$. pylori-infected and -eradicated cases. The NBI/BLIand $\mathrm{C}$-indices calculated by the image analysis may facilitate identification of small depressed-type GC.

\section{Introduction}

It has been postulated that Helicobacter pylori ( $H$. pylori) infection causes gastric cancer (GC) [1]. In 2013, in order to prevent the development of GC, the national health insurance program in Japan began to cover eradication therapy for patients with endoscopically diagnosed chronic gastritis caused by $\mathrm{H}$. pylori infection [2]. Similarly, in 2014 the International Agency for Research on Cancer Working Group Report recommended that all countries explore the possibility of introducing populationbased $H$. pylori screening and treatment programs as a strategy for GC prevention [3]. However, long-term studies from Japan showed that eradication of $H$. pylori does not substantially alter the risk of development of GC $[4,5]$. 
Based on many studies, it is evident that magnifying narrowband imaging (NBI) is useful for the accurate diagnosis of GCseven for small, depressed-type intramucosal GCs [6-9]. It was reported that the macroscopic appearance of gastric neoplasms, including GC and adenoma, was altered by $H$. pylori eradication therapy $[10,11]$. Several research groups subsequently reported that GCs are difficult to diagnose after $H$. pylori eradication even by magnifying NBI endoscopy because of their indistinct borders and/or lack of obviously cancerous characteristics [12-15]. The reason for this may be because an epithelium with low grade atypia or a non-neoplastic epithelium on GC tissue frequently covers the surface; in addition, the surface structure was found to have a "gastritis-like" appearance in $44 \%$ of a series of GCs after $H$. pylori eradication [12]. The features of GC detected after successful eradication therapy were as follows: small lesion size, location in the non-cardiac part of the stomach, a depressed type, and differentiated histology [16].

It was reported recently that magnifying blue laser imaging (BLI), a novel image-enhanced endoscopy (IEE) modality, provided a better diagnostic performance for early GC than conventional endoscopy with white-light imaging, and the diagnostic effectiveness of magnifying BLI was described as similar to that of magnifying NBI [17]. However, although endoscopic systems equipped with $\mathrm{NBI}$ and BLI are widely used, only a limited number of cases are subjected to magnifying $\mathrm{NBI} / \mathrm{BLI}$ endoscopy, even in hospitals specializing in gastroenterology. Therefore, many gastroenterologists and endoscopists may be using a conventional non-magnifying (NM)-NBI/BLI endoscope, which lacks a magnification function, to screen for early GC. There is unfortunately no reported research on the conventional NM-NBI/BLI of small $(\leq 1 \mathrm{~cm})$ early GC. Therefore, the primary end point of this study was to retrospectively investigate NM-NBI/BLI findings to discriminate depressed-type GCs from benign depressed lesions (BDLs) in $H$. pylori-infected and -eradicated cases using an image analysis system. Secondary end points were (1) to analyze cutoff indices of NM-NBI/BLI findings for GC diagnosis, and (2) to elucidate the association between NBI/BLI findings and histological architectures in GCs.

\section{Materials and methods}

This study complied with the Standards for the Reporting of Diagnostic Accuracy Studies (STARD) initiative [18] and the Declaration of Helsinki. The Ethics Committee of Hyogo College of Medicine approved this study protocol (No.2532). Written informed consent was obtained from all patients who underwent an endoscopic procedure.

\section{Patients}

Between April 2013 and March 2017, 346 consecutive patients with a total of 403 early GCs were treated with endoscopic submucosal dissection (ESD) at Hyogo College of Medicine Hospital. Among these patients, we enrolled those with images of (1) small $(\leq 1 \mathrm{~cm})$ depressed-type intramucosal GCs, (2) histologically differentiated-type GCs, and (3) patients whose $H$. pylori status had been confirmed. Patients with multiple GCs were excluded. > Fig. 1 shows a flow chart of the study patients. As can

\begin{tabular}{|c|c|}
\hline \multicolumn{2}{|c|}{ Early gastric cancer $(n=403)$} \\
\hline \multicolumn{2}{|c|}{ Submucosal gastric cancer $(n=60)$} \\
\hline \multicolumn{2}{|c|}{ Mucosal gastric cancer $(n=343)$} \\
\hline \multicolumn{2}{|c|}{$\begin{array}{l}\text { Excluded }(\mathbf{n}=\mathbf{2 5 3}) \\
\text { - }>10 \mathrm{~mm}(\mathrm{n}=175) \\
\text { - type I and Ila }(n=52) \\
\text { - multiple gastric cancers }(n=4) \\
\text { - } \text { with ulcer scar }(n=8) \\
\text { - undifferentiated-type adenocarcinoma }(n=14)\end{array}$} \\
\hline \multicolumn{2}{|c|}{ Eligible lesions ( $n=90)$} \\
\hline \multicolumn{2}{|c|}{$\begin{array}{l}\text { Excluded }(\mathbf{n}=\mathbf{6 1}) \\
\text { - H. pylori uninfected }(n=7) \\
\text { - naturally eradicated }(n=7) \\
\text { - lesion with biopsied previously, bleeding or white } \\
\text { coating }(n=47)\end{array}$} \\
\hline & $\downarrow$ \\
\hline $\begin{array}{l}\text { H. pylori infected } \\
(n=15)\end{array}$ & $\begin{array}{l}\text { H. pylori eradicated } \\
(n=14)\end{array}$ \\
\hline
\end{tabular}

- Fig. 1 Flow chart of the study patients with early gastric cancer.

be seen, 29 consecutive GC cases (the GC group) were evaluated. In addition, among the 15713 patients who had been endoscopically diagnosed as having chronic atrophic gastritis during the same period at the same hospital, 166 patients with BDLs (the BDL group, i.e. the control group) whose $H$. pylori status had been confirmed were selected.

In the BDL group, 47 lesions were positive for $H$. pylori and 90 were eradicated. The BDLs, i. e. depressed erosions, raised erosions, and areas of patchy redness, were endoscopically defined based on the Kyoto Classification of Gastritis [19] and were confirmed histologically as benign lesions. Lesions that underwent a biopsy before the detection of GC or BDLs were excluded because a biopsy causes morphological and color changes of the lesions due to the regenerative reaction. Lesions with bleeding or with a white coating were also excluded, because these conditions make the accurate judgment of IEE images difficult.

\section{Endoscopy procedure and histology}

All individuals underwent $\mathrm{NBI}$ or BLI endoscopy (endoscopes: GIF-Q260, H260, H260Z, H290, HQ290 or H290Z; EG-L590WR or -L600ZW) with an electronic endoscopic system (Elite CV290: Olympus Medical Systems, Tokyo; LASEREO: Fujifilm Holdings, Tokyo). The histology, macroscopic type, and depth of invasion in the resected materials obtained by ESD and biopsy specimens fulfilled the gastric cancer criteria of the Japanese Gastric Cancer Association [20]. 


\section{Image analysis}

To assess the NM-NBI/BLI findings objectively, the NBI/BLI of the GCs and BDLs that underwent ESD or a biopsy was quantified with an image-analytical software program as described previously [21]. Briefly, NBI/BLI images were converted into Joint Photographic Expert Group images, and then the GC and BDL areas on the images were manually traced with an image-analytical software program (Image), ver. 1.48; U.S. National Institutes of Health, Bethesda, MD, United States). Using the default method, i.e. the "Measure" tool in the "Analyze" menu, the mean gray value (MGV) of the lesion was calculated, and the MGV of the perilesional normal mucosa, which corresponds to the peripheral elevation surrounding the depressed area, was also measured. The ratio of the MGV of the GC or BDL areas against that of the perilesional areas was defined as the NBI/ BLI index ( $>$ Fig. 2; note, a brighter image has a higher NBI/BLIindex).

In addition, the values for shape descriptors of the GCs and BDLs were also calculated for assessment of their shapes. Briefly, using the default tool "Measure" under the "Analyze" menu, "Circ." was adopted as the circularity index (C-index). The C-index value of a perfect circle is 1 ; as the shape deviates from perfectly circular, the $\mathrm{C}$-index value decreases ( $>$ Fig. 2 ). The C-index thus indicates an overall irregular shape for an entire lesion, but not local irregularities of the lesion edge. This quantification was performed by a laboratory assistant (C.I.) who did not have any medical knowledge about endoscopies or GC, and did not have any information on the patients' original diagnosis or treatment.

\section{Evaluation of histological architecture}

The histopathological images of GCs resected by ESD were evaluated using cellSens Standard software, ver. 1.8.1 (Olympus, Tokyo). In accordance with a previous report [22], we analyzed the characteristics of the histopathological findings. Namely, an endoscopist who was not aware of the information on the NBI/BLI-index randomly selected three areas of the intervening parts and crypts in the cancerous area, and measured the length of the intervening part and the depth of the crypt in each lesion. Briefly, the length of the intervening part was defined as the distance between the crypt and adjacent crypt. The depth of the crypt was defined as the distance between the bottom of the crypt and the line that connects the top of the crypt structure ( $\triangleright$ Fig. $\mathbf{3}$ ).

\section{Diagnosis of $\mathrm{H}$. pylori status}

The $H$. pylori status was analyzed in each patient by three methods: Giemsa staining, urea breath test, and measurement of the serum level of $H$. pylori-lgG antibodies with an enzyme-linked immunosorbent assay (ELISA) kit using the E plate test (Eiken Kagaku, Tokyo, Japan). A patient was regarded as positive for $H$. pylori if the result of at least one of these methods was positive.

We divided the patients into three groups by $H$. pylori status: current infection, past infection, and uninfected. We also subdivided the patients with past infection into two groups: those with successful $H$. pylori eradication and those with an unknown
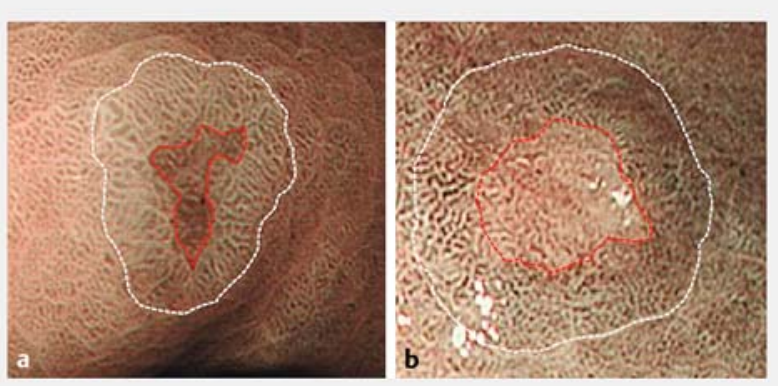

- Fig. 2 Image analysis of NBI/BLI- and C-indices. The mean gray value (MGV) was calculated in the depressed area surrounded by the red dotted line, and the MGV of the perilesional area was also determined in the region between the red dotted line and the white dotted line. a In this lesion diagnosed as a benign depressed lesion (BDL) by biopsy, the NBI/BLI- and C-indices were 0.80 and 0.40 , respectively. $\mathbf{b}$ In this gastric cancer case, the depressed area appears more brightly colored than the surrounding mucosa on magnifying endoscopy in the NBI/BLI mode. The NBI/BLI-index and $\mathrm{C}$-index were 1.30 and 0.92 , respectively, and thus higher than the values in the BDL.

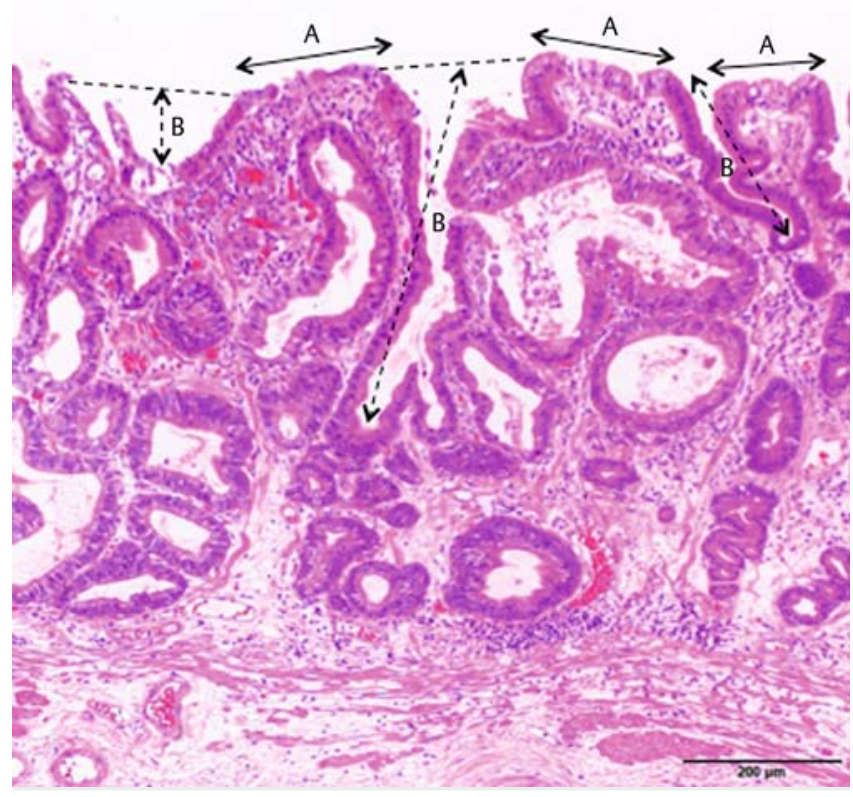

- Fig. 3 Measurement of the histological architecture. A, intervening parts; $\mathrm{B}$, crypt depth defined as the distance between the bottom of the crypt and the dotted line that connects the top of the crypt structures.

history of $H$. pylori eradication (naturally eradicated patients). $H$. pylori-uninfected patients were defined as those showing negative results in $\mathrm{H}$. pylori examinations and having no atrophic findings on endoscopy based on the Kyoto Classification of Gastritis [19]. In this study, we excluded the $H$. pylori-uninfected patients and the patients who were suspected of naturally eradicated $H$. pylori, since other high-sensitivity and high-specificity tests for $H$. pylori such as biopsy PCR and fluorescence in situ hybridization [23] were not performed, and thus $H$. pylori 
- Table 1 Clinical characteristics of the H. pylori-infected and -eradicated cases.

\begin{tabular}{|c|c|c|c|c|c|c|}
\hline & \multicolumn{3}{|c|}{ H. pylori-infected cases } & \multicolumn{3}{|c|}{ H. pylori-eradicated cases } \\
\hline & GC & BDLs & $P$ value & GC & BDLs & $P$ value \\
\hline No. of lesions & 15 & 47 & & 14 & 90 & \\
\hline Age, mean $\pm S D, y$ & $68.8 \pm 11.1$ & $63.9 \pm 15.1$ & 0.26 & $71.2 \pm 6.5$ & $67.6 \pm 9.4$ & 0.21 \\
\hline Female, no. (\%) & $3(20.0)$ & $11(28.6)$ & 0.74 & $3(21.4)$ & $26(34.2)$ & 0.37 \\
\hline Tumor size, mean $\pm S D, m m$ & $7.2 \pm 1.64$ & - & & $6.2 \pm 2.71$ & - & $0.84^{1}$ \\
\hline \multicolumn{7}{|l|}{ Location } \\
\hline - Upper third & 2 & 7 & 0.92 & 2 & 11 & 0.74 \\
\hline - Middle third & 6 & 16 & & 7 & 54 & \\
\hline - Lower third & 7 & 24 & & 5 & 25 & \\
\hline - Lesser curvature & 3 & 18 & 0.44 & 7 & 45 & 0.65 \\
\hline - Greater curvature & 8 & 16 & & 1 & 10 & \\
\hline - Anterior wall & 2 & 4 & & 2 & 5 & \\
\hline - Posterior wall & 2 & 9 & & 4 & 30 & \\
\hline
\end{tabular}

- Table 2 IEE indices in the H. pylori-infected and -eradicated cases.

\begin{tabular}{|l|l|l|l|l|l|l|}
\hline & \multicolumn{2}{|l|}{ H. pylori-infected cases } & \multicolumn{3}{l|}{ H. pylori-eradicated cases } \\
\hline & GC & BDLs & P value & GC & BDLs \\
\hline NBI/BLI-index & $1.03 \pm 0.19$ & $0.89 \pm 0.11$ & 0.009 & $1.06 \pm 0.13$ & $0.87 \pm 0.10$ \\
\hline C-index & $0.66 \pm 0.18$ & $0.52 \pm 0.15$ & 0.006 & $0.67 \pm 0.19$ & $0.49 \pm 0.17$ \\
\hline IEE, image-enhanced endoscopy; GC, gastric cancer; BDLs, benign depressed lesions. & & 0.004 \\
\hline
\end{tabular}

infection could not be completely ruled out in these individuals. We analyzed the images of GC and BDLs only in patients with current infection of $H$. pylori or with successful eradication of H. pylori.

\section{Statistical analysis}

Continuous and categorical data were reported as means and standard deviations (SDs). The data were assessed by the Mann-Whitney $U$ test for continuous variables and the chisquared test or Fisher's exact test for categorical variables between two independent groups. To identify the NBI/BLI- and $\mathrm{C}$-indices indicating the highest diagnostic performance for GC, we performed a receiver operating characteristic (ROC) curve analysis. The area under the ROC curve (AUC) was calculated, and the point with the largest AUC was defined as the point that had the greatest association with GC. Optimal cutoff points were determined on the basis of the maximum values of the Youden index, calculated as [sensitivity + specificity -1 ], and the minimum values of the square root of $\left[(1-\text { sensitivity })^{2}\right.$ $\left.+(1 \text { - specificity })^{2}\right]$, which indicates the minimum distance from the upper left corner to the point on the ROC curve [24]. In the current study, we used the ROC curve analysis to evaluate the efficiency of the differential diagnosis between GC and BDLs by NBI/BLI- and C-indices in the $\mathrm{H}$. pylori-infected and -eradicated cases. We used SPSS ver. 22.0 software (IBM Corp., Armonk, NY, United States) or GraphPad Prism5 (GraphPad Software, La Jolla, CA, United States) for all analyses. Two-tailed $P$ values less than 0.05 were considered to be statistically significant.

\section{Results}

\section{Patients}

- Table 1 summarizes the clinical characteristics of the cases. There were no statistically significant differences in the mean age, gender ratio, or lesion location of patients between GC and BDLs in either the $H$. pylori-infected or -eradicated cases.

\section{$\mathrm{NBI} / \mathrm{BLI}$ - and $\mathrm{C}$-indices in the GC and BDL cases}

The mean NBI/BLI- and C-indices were significantly higher in the GC group than in the BDL group, in both $H$. pylori-infected and -eradicated cases $(P=0.009$ in GC and $P<0.0001$ in BDLs for the NBI/BLI-index; $P=0.006$ in GC and $P=0.004$ in BDLs for the C-index) ( $>$ Table 2 ). These results indicate that GCs are brighter than the surrounding mucosa and rounder in shape 


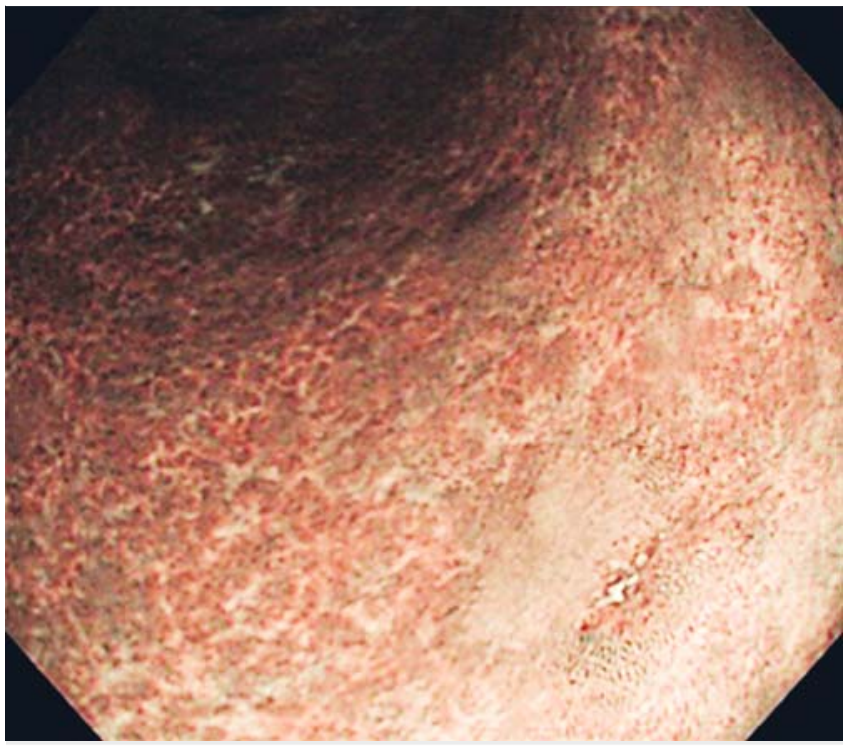

- Fig. 4 Representative non-magnifying NBI image of H. pylori-infected gastric cancer. The depressed area is brighter than the surrounding area. The NBI/BLI-index and C-index were 1.11 and 0.81 , respectively.

than BDLs in both the $H$. pylori-infected and -eradicated cases ( $\triangleright$ Fig. 1, $\triangleright$ Fig. 4, and $\triangleright$ Fig. 5).

\section{Association between the $\mathrm{NBI} / \mathrm{BLI}$-index or C-index and GC or BDLs}

The ROC curve for the differential diagnosis between GC and BDLs by the NBI/BLI- and C-indices in the $H$. pylori-infected and -eradicated cases is shown in $\mathbf{F i g . 6}$. The resulting cutoff value of the $\mathrm{NBI} / \mathrm{BLI}$-index was 1.04 (AUC $=0.72$, sensitivity $60.0 \%$, specificity $89.4 \%$, and $P=0.0001)$ and that of the $C$-index was 0.58 ( $A \cup C=0.74$, sensitivity $80.0 \%$, and specificity $66.0 \%$ ) for GC in the $H$. pylori-infected cases. In the $H$. pylori-eradicated cases, the thresholds for GC were 0.98 in the $\mathrm{NBI} / \mathrm{BLI}$-index ( $A \cup C=0.91$, sensitivity $92.9 \%$, and specificity $92.2 \%$ ) and 0.64 in the $\mathrm{C}$-index $(\mathrm{AUC}=0.74$, sensitivity $71.4 \%$, and specificity $77.8 \%$ ) ( Table 3 ). The NBI/BLI and C-indices, which were $\geq 1.04$ and $\geq 0.58$ in the $H$. pylori-infected cases, and $\geq 0.98$ and $\geq 0.64$ in the $H$. pylori-eradicated cases, were significant predictors of GCs (odds ratio $[\mathrm{OR}]=69.0,95 \%$ confidence interval $[\mathrm{Cl}]$ $3.57-17.03, P<0.0001$ in the $H$. pylori-infected cases; $O R=$ 52.2, $95 \% \mathrm{Cl} 5.54-34.39, P<0.0001$ in the $H$. pylori-eradicated cases).

\section{Diagnostic accuracy at various thresholds of the $\mathrm{NBI} / \mathrm{BLI}$ - and C-indices in the $\mathrm{H}$. pylori-infected and -eradicated cases}

In 2012, the American Society for Gastrointestinal Endoscopy (ASGE) established recommended thresholds for the Preservation and Incorporation of Valuable Endoscopic Innovations (PIVI) to eliminate the need for random mucosal biopsies during the endoscopic surveillance of patients with a non-dysplastic Barrett's esophagus. According to the PIVI document released by the ASGE, the recommended performance thresholds

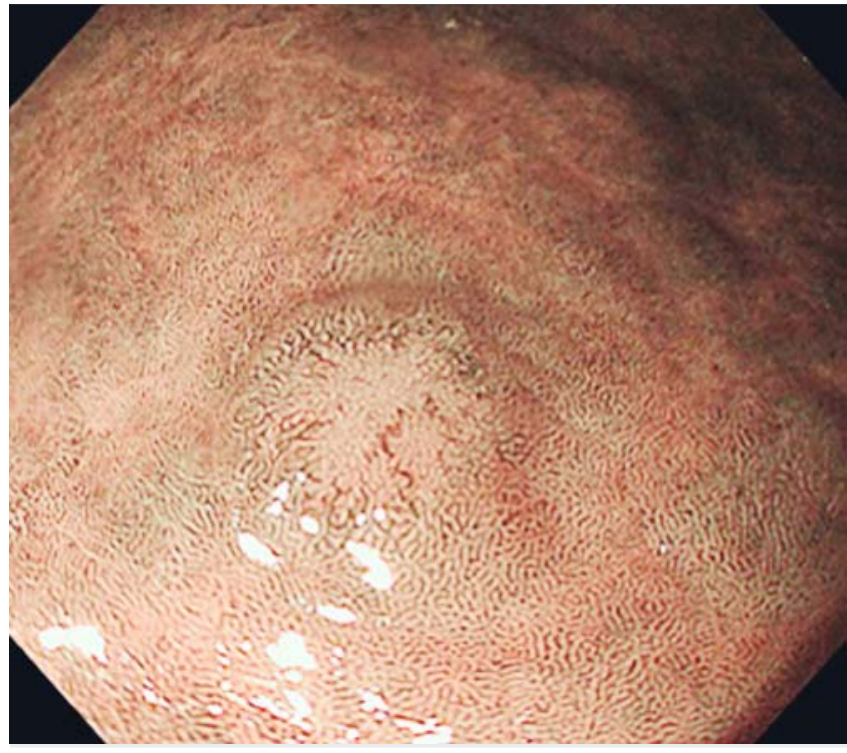

- Fig. 5 Representative gastric cancer case after $H$. pylori eradication. The depressed area is a relatively bright area compared to the surrounding area. The NBI/BLI-index and C-index were 1.26 and 0.86 , respectively.

were as follows: imaging technology with targeted biopsies should have a per-patient sensitivity of $\geq 90 \%$ and a negative predictive value (NPV) of $\geq 98 \%$ for detecting high grade dysplasia or early esophageal adenocarcinoma, compared with the current standard protocol [25]. Thus, based on these thresholds - because there is no PIVI document with regard to GC - we analyzed the diagnostic accuracy of GC by applying a few useful cutoff points of the NBI/BLI- and C-indices as diagnostic parameters for GC ( $\triangleright$ Table 4). When we set the reference values of the NBI/BLI-index for GC at $\geq 0.69$ with the $C$-index at $\geq 0.21$ in the $H$. pylori-infected cases, and the values of the NBI/BLI-index at $\geq 0.80$ with the $\mathrm{C}$-index at $\geq 0.32$ in the $H$. pylori-eradicated cases, the sensitivity and NPV for early GC were both $100 \%$.

\section{Relationship between NBI/BLI-index and histological architecture}

From the group of patients with GC who underwent resection by ESD, we selected 20 resected specimens for which the histological architecture could be correctly examined in sagittal sections. The length of the intervening part and the depth of crypts ( $\triangleright$ Fig. $\mathbf{3}$ ) did not show a significant difference in cutoff points of the NBI/BLI-index between $<1.04$ and $\geq 1.04$ in the $H$. pylori-infected cases ( $\triangleright$ Table 5 ). In the $H$. pylori-eradicated cases, however, the length of the intervening parts tended to be wider in lesions with $\geq 0.98$ as the cutoff point compared to those with $<0.98$ as the cutoff point $(P=0.09)$, but the depth of crypts was not significantly different. 

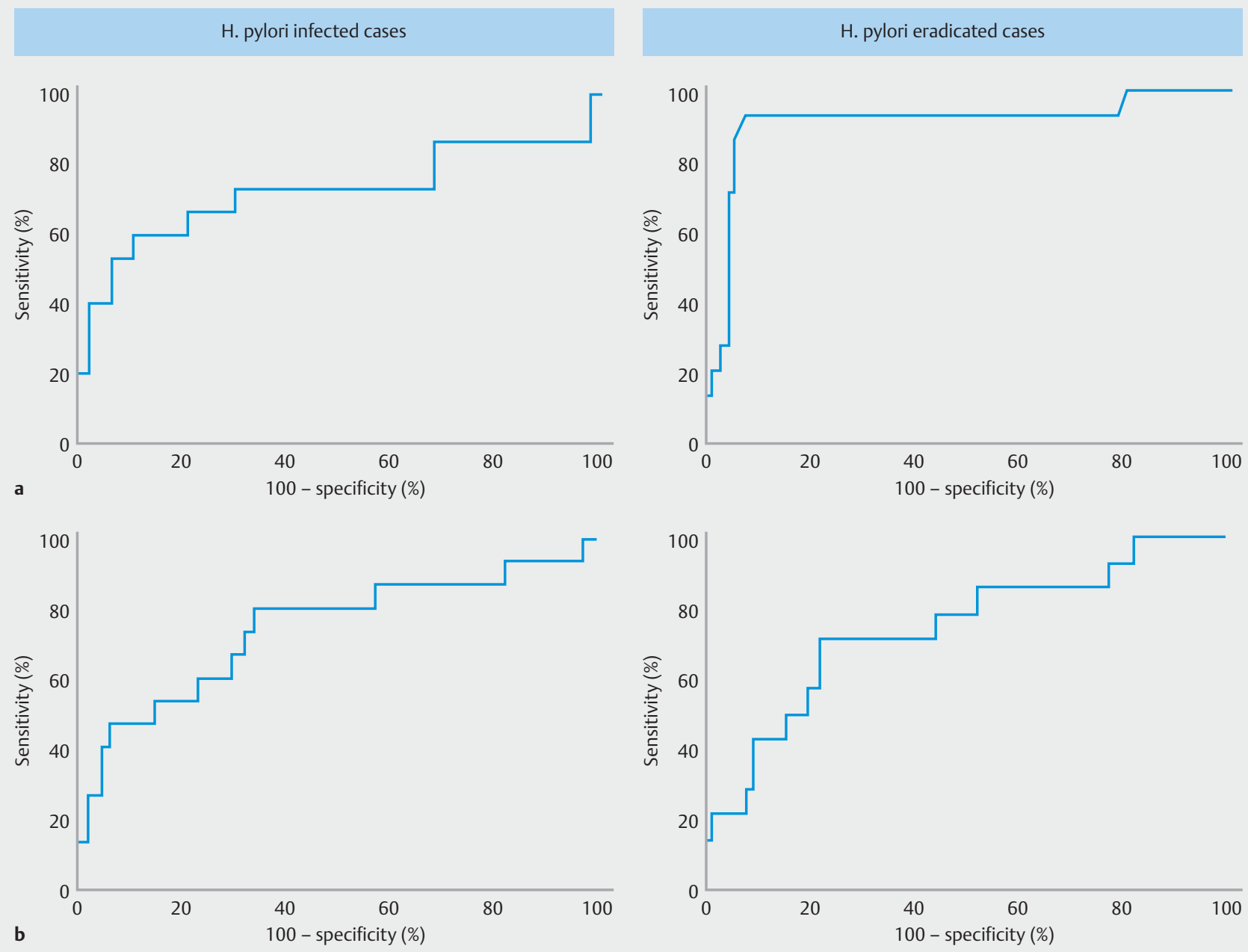

Fig. 6 Receiver operating characteristic curve of the NBI/BLI-index (a) and C-index (b) values of gastric cancer in the $H$. pylori-positive cases and H. pylori-eradicated cases. The curve is plotted as the sensitivity ( $y$-axis) and ( 100 - specificity) ( $x$-axis).

\section{Discussion}

GC after $H$. pylori eradication is often difficult to diagnose, even by magnifying $\mathrm{NBI} / \mathrm{BLI}$ endoscopy [11-15]. However, the present study clearly demonstrated the possibility of detecting small depressed-type early GCS in both $H$. pylori-infected and -eradicated cases by NM-NBI/BLI endoscopy with objective indicators.

Several recent studies reported the potential quantitative diagnosis of early GC by computer-aided/computer-based diagnosis and deep learning [26-29]. However, those studies investigated the identification and delineation of early GCs using magnifying NBI or BLI, and they did not suggest a region of interest during NM-endoscopy, nor did they attempt to capture GCs during such screening NM-endoscopy using IEE [26-29].

Our present analyses revealed that both NBI/BLI- and C-indices calculated using NM-NBI/BLI images were significantly higher in the GC group than in the BDL group in both the $H$. pylori-infected and -eradicated cases, indicating that (1) the depressed area in GCs is a relatively bright color against the color of the surrounding perilesional area, and (2) GCs are rounder in shape than BDLs. However, the AUC of the NBI/BLI-index was sufficiently higher in the $H$. pylori-eradicated cases $(0.91)$ than in the $H$. pylori-infected cases (0.72). Like the NBI/BLI-index, the $\mathrm{C}$-index also showed a high accuracy rate for GC diagnosis in the $\mathrm{H}$. pylori-eradicated cases $(76.9 \%)$ when the appropriate cutoff point was applied. Commonly, early depressed-type GC, especially differentiated-type adenocarcinoma, exhibits a reddish and irregularly shaped depression [30,31]. Although the irregularity, i.e. the spine-like appearance, at the edge of the depression is a predictive indicator of depressed GC, the C-index evaluated in our study indicates the irregular shape of the entire lesion rather than just its edge.

Based on the established PIVI thresholds for imaging in Barrett's esophagus [25], lesions with high sensitivity and a high NPV (such as the NBI/BLI-index of $\geq 0.69$ with the C-index at $\geq 0.21$ in the $H$. pylori-infected cases and the NBI/BLI-index of $\geq 0.80$ with the $\mathrm{C}$-index at $\geq 0.32$ in the $H$. pylori-eradicated cases) should be biopsied in order to compensate for low specificity $(4.3-34.4 \%)$ and accuracy $(27.4-43.3 \%)$. Since the diag- 
- Table 3 Parameters of the ROC curves for gastric cancer diagnosis.

\begin{tabular}{|c|c|c|c|c|}
\hline & \multicolumn{2}{|c|}{ H. pylori-infected cases } & \multicolumn{2}{|c|}{ H. pylori-eradicated cases } \\
\hline & NBI/BLI-index & C-index & NBI/BLI-index & C-index \\
\hline Cutoff point & 1.04 & 0.58 & 0.98 & 0.64 \\
\hline AUC & 0.72 & 0.74 & 0.91 & 0.74 \\
\hline SE & 0.09 & 0.08 & 0.05 & 0.08 \\
\hline $95 \% \mathrm{Cl}$ & $0.54-0.91$ & $0.58-0.90$ & $0.81-1.02$ & $0.59-0.89$ \\
\hline$P$ value & 0.009 & 0.006 & $<0.0001$ & 0.004 \\
\hline Sensitivity (\%) & 60.0 & 80.0 & 92.9 & 71.4 \\
\hline Specificity (\%) & 89.4 & 66.0 & 92.2 & 77.8 \\
\hline PPV (\%) & 64.3 & 42.9 & 65.0 & 33.3 \\
\hline NPV (\%) & 87.5 & 91.2 & 98.8 & 94.6 \\
\hline \multirow[t]{2}{*}{ Accuracy (\%) } & 82.3 & 69.4 & 92.3 & 76.9 \\
\hline & \multicolumn{2}{|c|}{ OR $69.0,95 \% \mathrm{Cl} 3.57-17.03, P<0.0001$} & \multicolumn{2}{|c|}{ OR 52.2, $95 \%$ CI 5.54-34.39, $P<0.0001$} \\
\hline
\end{tabular}

ROC, receiver operating characteristic; AUC, area under the ROC curve; SE, standard error; Cl, confidence interval; PPV, positive predictive value; NPV, negative predictive value; $O R$, odds ratio.

Table 4 Diagnostic values for gastric cancer using the criteria of the NBI/BLI-index and C-index.

\begin{tabular}{|c|c|c|c|c|c|c|}
\hline \multicolumn{7}{|c|}{ Diagnostic parameters for GC } \\
\hline NBI/BLI-index & C-index & Sensitivity (\%) & Specificity (\%) & PPV (\%) & NPV (\%) & Accuracy (\%) \\
\hline \multicolumn{7}{|c|}{ H. pylori-infected cases } \\
\hline$(\geq 0.69)$ & $(\geq 0.21)$ & & & & & \\
\hline+ & + & 100 & 4.3 & 25.0 & 100 & 27.4 \\
\hline+ & - & 0 & 97.9 & 0 & 75.4 & 74.2 \\
\hline- & + & 0 & 97.9 & 0 & 75.4 & 74.2 \\
\hline$(\geq 0.69)$ & $(\geq 0.38)$ & & & & & \\
\hline+ & + & 86.7 & 19.1 & 25.5 & 81.8 & 35.5 \\
\hline+ & - & 6.7 & 83.0 & 11.1 & 73.6 & 64.5 \\
\hline- & + & 6.7 & 97.9 & 50.0 & 76.7 & 75.8 \\
\hline \multicolumn{7}{|c|}{ H. pylori-eradicated cases } \\
\hline$(\geq 0.80)$ & $(\geq 0.32)$ & & & & & \\
\hline+ & + & 100 & 34.4 & 19.2 & 100 & 43.3 \\
\hline+ & - & 0 & 85.6 & 0 & 84.6 & 74.0 \\
\hline- & + & 0 & 83.3 & 0 & 84.3 & 72.1 \\
\hline$(\geq 0.98)$ & $(\geq 0.64)$ & & & & & \\
\hline+ & + & 64.3 & 96.7 & 75.0 & 94.6 & 92.3 \\
\hline+ & - & 28.6 & 95.6 & 50.0 & 89.6 & 86.5 \\
\hline- & + & 7.1 & 81.1 & 5.6 & 84.9 & 71.2 \\
\hline
\end{tabular}


- Table 5 Correspondence between the NBI/BLI-index and histological architecture in gastric cancer.

\begin{tabular}{|c|c|c|c|c|c|c|}
\hline & \multicolumn{3}{|c|}{ H. pylori-infected case $(n=9)$} & \multicolumn{3}{|c|}{ H. pylori-eradicated case $(n=11)$} \\
\hline & \multicolumn{3}{|l|}{ Cutoff point } & \multicolumn{3}{|l|}{ Cutoff point } \\
\hline & $<1.04$ & $\geq 1.04$ & $P$ value & $<0.98$ & $\geq 0.98$ & $P$ value \\
\hline Length of intervening parts & $192.7 \pm 153.0$ & $183.9 \pm 65.2$ & 0.49 & $130.7 \pm 93.4$ & $197.3 \pm 97.6$ & 0.09 \\
\hline Depth of crypts & $201.4 \pm 110.2$ & $195.9 \pm 89.2$ & 0.85 & $121.1 \pm 37.8$ & $120.7 \pm 63.6$ & 0.90 \\
\hline
\end{tabular}

nosis of early GC after successful $H$. pylori eradication is not necessarily satisfactory even by magnifying endoscopy with $\mathrm{NBI} /$ BLI [12-15], our diagnostic strategy may be appropriate. Although it is undeniable that magnifying NBI/BLI is an important modality for finding the irregular microvascular and microsurface patterns in the diagnosis of GC [6-9], the NBI/BLI-index calculated by NM-NBI/BLI endoscopy reflects a "mixed whole image" of microvascular and microsurface features. In daily endoscopy, we often find depressed lesions, and occasionally multiple depressed lesions. Therefore, our objective data may provide useful information on the future use of artificial intelligence (AI), including computer-aided/computer-based diagnosis and deep learning, for the detection and diagnosis of GC.

Interestingly, the bright color image of the depressed area compared to the surrounding perilesional color, which corresponds to an NBI/BLI-index $\geq 0.98$ derived from the ROC analysis, was associated with a greater length of the intervening part in the $H$. pylori-eradicated cases, although this relationship did not reach the level of statistical significance. This trend of association might be ascribed to the fact that, in cases of differentiated-type GC after $H$. pylori eradication, an epithelium with low grade atypia or non-neoplastic epithelium covers the GC tissue [11-15]. However, we observed no significant difference in the association between the length of the intervening part and the $\mathrm{NBI} / \mathrm{BLI}$-index in the $\mathrm{H}$. pylori-infected cases in the present study. Therefore, the infiltration of inflammatory cells in the cancerous mucosa in the $H$. pylori-infected cases may have contributed to the difference in the NBI/BLI findings in addition to the length of the intervening part. In contrast, the depth of crypts was not involved in the color difference in either the $H$. pylori-infected or -eradicated cases.

The present study had a methodological advantage, as all $\mathrm{NBI} / \mathrm{BLI}$ - and C-indices were evaluated by a single laboratory assistant (C.I.) who did not have endoscopy expertise and was unaware of the lesion groups. Therefore, the proposed method could lead to future Al research for early GC diagnosis. However, our investigation also had some limitations. First, this was a study from a single institution with a small number of cases. Second, because all BDLs could not be selected during the study period, the BDL group was vulnerable to selection bias, while there were no significant differences in the clinical characteristics, including the sex ratio, mean age, lesion location, and $H$. pylori status, between GC and BDLs. Third, it was not possible to examine all lesions by both modalities, i.e. NM$\mathrm{NBI}$ and -BLI endoscopy. However, when we analyzed the "ratio" of the MGV of the GC or BDL areas against the perilesional areas by $\mathrm{NBI}$ or BLI, the difference in modality did not appear to substantially affect the results of the NBI/BLI-index. Fourth, the endoscopic images of the GCs and BDLs were selected by experienced endoscopists and were reviewed retrospectively. To overcome these limitations, further investigations with larger sample sizes including differentiated-type early GCs and BDLs are needed to validate our endoscopic findings and the objective indicators, followed by a multicenter and prospective study to exclude selection bias.

In conclusion, the brighter color and rounder shape of small depressed lesions determined by the NBI/BLI- and C-indices in non-magnifying IEE images may increase the index of suspicion of early GC in both $H$. pylori-infected and -eradicated patients, thus these lesions should be biopsied for histological diagnosis.

\section{Competing interests}

None

References

[1] Correa P. Helicobacter pylori and gastric carcinogenesis. Am J Surg Pathol 1995; 19: S37-S43

[2] Asaka M, Kato M, Sakamoto N. Roadmap to eliminate gastric cancer with Helicobacter pylori eradication and consecutive surveillance in Japan. J Gastroenterol 2014; 49: 1 -8

[3] IARC Helicobacter pylori Working Group. Helicobacter pylori eradication as a strategy for preventing gastric cancer. IARC Working Group Reports, Vol. 8 Lyons, France: International Agency for Research on Cancer; 2014

[4] Take S, Mizuno M, Ishiki K et al. The long-term risk of gastric cancer after the successful eradication of Helicobacter pylori. J Gastroenterol 2011; 46: 318-324

[5] Kodama M, Murakami K, Okimoto T et al. Histological characteristics of gastric mucosa prior to Helicobacter pylori eradication may predict gastric cancer. Scand J Gastroenterol 2013; 48: 1249-1256

[6] Nakayoshi T, Tajiri H, Matsuda K et al. Magnifying endoscopy combined with narrow band imaging system for early gastric cancer: correlation of vascular pattern with histopathology (including video). Endoscopy 2004; 36: 1080 - 1084

[7] Yao K, Anagnostopoulos GK, Ragunath K. Magnifying endoscopy for diagnosing and delineating early gastric cancer. Endoscopy 2009; 41: $462-467$

[8] Ezoe $\mathrm{Y}$, Muto $\mathrm{M}$, Uedo $\mathrm{N}$ et al. Magnifying narrowband imaging is more accurate than conventional white-light imaging in diagnosis of gastric mucosal cancer. Gastroenterology 2011; 141: 2017-2025 
[9] Li HY, Dai ], Xue HB et al. Application of magnifying endoscopy with narrow-band imaging in diagnosing gastric lesions: a prospective study. Gastrointest Endosc 2012; 76: 1124-1132

[10] Gotoda T, Saito D, Kondo H et al. Endoscopic and histological reversibility of gastric adenoma after eradication of Helicobacter pylori. J Gastroenterol 1999; 34: (Suppl. 11): 91 - 96

[11] Ito M, Tanaka S, Takata S et al. Morphological changes in human gastric tumours after eradication therapy of Helicobacter pylori in a short-term follow-up. Aliment Pharmacol Ther 2005; 21: 559-566

[12] Kobayashi M, Hashimoto S, Nishikura K et al. Magnifying narrow-band imaging of surface maturation in early differentiated-type gastric cancers after Helicobacter pylori eradication. J Gastroenterol 2013; 48: $1332-1342$

[13] Kitamura Y, Ito M, Matsuo T et al. Characteristic epithelium with lowgrade atypia appears on the surface of gastric cancer after successful Helicobacter pylori eradication therapy. Helicobacter 2014; 19: 289 295

[14] Hori K, Watari J, Yamasaki T et al. Morphological characteristics of early gastric neoplasms detected after Helicobacter pylori eradication. Dig Dis Sci 2016; 61: 1641 - 1651

[15] Saka A, Yagi K, Nimura S. Endoscopic and histological features of gastric cancers after successful Helicobacter pylori eradication therapy. Gastric Cancer 2016; 19: 524-530

[16] Kamada T, Hata J, Sugiu K et al. Clinical features of gastric cancer discovered after successful eradication of Helicobacter pylori: results from a 9-year prospective follow-up study in Japan. Aliment Pharmacol Ther 2005; 21: $1121-1126$

[17] Dohi O, Yagi N, Majima A et al. Diagnostic ability of magnifying endoscopy with blue laser imaging for early gastric cancer: a prospective study. Gastric Cancer 2017; 20: 297 -303

[18] Bossuyt PM, Reitsma JB, Bruns DE et al. STARD Group. STARD 2015: an updated list of essential items for reporting diagnostic accuracy studies. BMJ 2015; 351: h5527

[19] Haruma K, Kato M, Inoue K et al. Kyoto classification of gastritis. Tokyo: Nihon Medical Center; 2014

[20] Japanese Gastric Cancer Association. Japanese classification of gastric carcinoma - 3rd English edn. Gastric Cancer 2011; 14: 101 - 112
[21] Watari ], Tomita T, Ikehara $\mathrm{H}$ et al. Diagnosis of small intramucosal signet ring cell carcinoma of the stomach by non-magnifying narrowband imaging: A pilot study. World J Gastrointest Endosc 2015; 7: $1070-1077$

[22] Yagi K, Nozawa Y, Endou S et al. Diagnosis of early gastric cancer by magnifying endoscopy with NBI from viewpoint of histological imaging: mucosal patterning in terms of white zone visibility and its relationship to histology. Diagn Ther Endosc 2012; 2012: 954809

[23] Aktepe OC, Ciftçi IH, Safak B et al. Five methods for detection of Helicobacter pylori in the Turkish population. World J Gastroenterol 2011; 17: $5172-5176$

[24] Akobeng AK. Understanding diagnostic tests 3: Receiver operating characteristic curves. Acta Paediatr 2007; 96: 644-647

[25] Sharma P, Savides T], Canto MI et al. The American Society for Gastrointestinal Endoscopy PIVI (Preservation and Incorporation of Valuable Endoscopic Innovations) on imaging in Barrett's esophagus. Gastrointest Endosc 2012; 76: 252-254

[26] Miyaki R, Yoshida S, Tanaka S et al. A computer system to be used with laser-based endoscopy for quantitative diagnosis of early gastric cancer. J Clin Gastroenterol 2015; 49: 108-115

[27] Liu X, Wang C, Bai J et al. Hue-texture-embedded region-based model for magnifying endoscopy with narrow-band imaging image segmentation based on visual features. Comput Methods Programs Biomed 2017; 145: 53-66

[28] Kanesaka T, Lee TC, Uedo N et al. Computer-aided diagnosis for identifying and delineating early gastric cancers in magnifying narrow-band imaging. Gastrointest Endosc 2018; 87: 1339-1344

[29] Hirasawa T, Aoyama K, Tanimoto T et al. Application of artificial intelligence using a convolutional neural network for detecting gastric cancer in endoscopic images. Gastric Cancer 2018; 21: 653-660

[30] Nakamura K. Structure of the gastric cancer. 3rd edn. Tokyo: Igakushoin; 2005: (in Japanese)

[31] Moriyama T, Akiyoshi D, Torisu T et al. Endoscopic findings of "former early gastric cancer" and "current early gastric cancer". Early Gastric Cancer 2018. Stomach Intestine 2018; 53: 534-544. Tokyo: IgakuShoin (in Japanese) 